\title{
SVN Isaneres \\ Effect of ischaemic brain injury on sexual function in adult mice
}

To cite: Tang Y, Yuan F, Cai $B$, et al. Effect of ischaemic brain injury on sexual function in adult mice. Stroke and Vascular Neurology 2016;1:e00013. doi:10.1136/svn-2016000013

Received 7 March 2016 Revised 30 June 2016 Accepted 4 July 2016 Published Online First 29 August 2016
CrossMark

\author{
${ }^{1}$ Neuroscience and \\ Neuroengineering Center, \\ Med-X Research Institute \\ Shanghai Jiao Tong \\ University, Shanghai, China \\ ${ }^{2}$ Shanghai Ruijin Hospital, \\ School of Medicine, \\ Shanghai Jiao Tong \\ University, Shanghai, China
}

Correspondence to Dr Guo-Yuan Yang; gyyang0626@163.com

\author{
Yaohui Tang, ${ }^{1}$ Falei Yuan, ${ }^{1}$ Beibei Cai, ${ }^{1}$ Weiliang Xia, ${ }^{1}$ Yongting Wang, \\ Guo-Yuan Yang ${ }^{1,2}$
}

\section{ABSTRACT \\ Objective: Priapism refers to a condition with persistent abnormal erection of the penis, which is usually caused by disease or injury in the brain or spinal cord, or obstruction to the outflow of blood through the dorsal vein at the root of the penis, without sexual desires. The effect of cerebral ischaemia on sexual function is unknown. The aim of this study is to explore whether priapism occurs in adult mice. Furthermore, we examined the relationship between priapism and the region of infarct in the brain. \\ Design: Adult male CD-1 mice who underwent permanent middle cerebral artery occlusion ( $\mathrm{PMCAO}$ ) were closely examined from 2 hours to 14 days postoperation.}

Results: We found that priapism occurs in $\sim 80 \%$ of the mice with pMCA0, which could persist up to 14 days. Further study has demonstrated that the occurrence of priapism is related to the infarct region: priapism is found only in mice with ischaemic injury extending to the hypothalamus and the hippocampus regions.

Conclusion: Our result suggested priapism may be used as a deep brain injury marker for evaluating brain injury in mice after pMCAO.

\section{INTRODUCTION}

Stroke is one of the leading causes of disability and mortality in the ageing population worldwide. ${ }^{1}$ Ischaemia or hypoxic brain injury often results in irreversible brain damage, mediated by multiple mechanisms including oxidative stress, inflammatory response, apoptosis, neuronal death, etc. ${ }^{2-4}$ Priapism is a persistent, painful penile erection, which can last for hours in the absence of sexual stimulation. It is found in $40 \%$ of patients with sickle cell disease, because of impaired drainage of the corpora cavernosa. ${ }^{5}$ Priapism often leads to erectile tissue fibrosis and ultimately results in erectile dysfunction. ${ }^{7}$ Several research groups studied the molecular mechanisms of priapism to obtain a better understanding of this disorder both experimentally and clinically. They found that opiorphin-induced priapism involves the activation of the polyamine synthetic pathway. ${ }^{8}$ Increased adenosine also contributes to penile fibrosis. ${ }^{9}$ Brain ischaemia-induced priapism is not well understood. Till now there are only a few case reports on brain injury induced priapism in clinic. Takaku et $a l^{10}$ reported a 35-year-old man had three attacks of subarachnoid haemorrhage in three consecutive years and suffered priapism with intolerable pain after the last attack of subarachnoid haemorrhage. Monga et $a l^{11}$ reported a 53-year-old man with an old temporal lobe infarction involving the anteromedial basal portion increase in libido and coital frequency with a tendency towards priapism. However, there is no animal model to mimic clinical priapism at present. In our group, we have consistently established middle cerebral artery occlusion (MCAO) models in mice as a stroke model. ${ }^{12-16}$ In this study, we report our observation that permanent MCAO (pMCAO) can induce priapism in adult mice. We explored the causative relationship between focal cerebral ischaemia and priapism occurrence. Priapism may serve as an indication for severe stroke damage in animal models.

\section{MATERIALS AND METHODS}

\section{Animal stroke model}

Animal procedures for the use of laboratory animals were approved by the Institutional Animal Care and Use Committee of Shanghai Jiao Tong University. Twenty-four adult male CD-1 mice (Shanghai SLAC Laboratory Animal CO) weighing 25-32 g were used. Focal cerebral ischaemia was conducted by pMCAO using the suture model as described previously. ${ }^{17}$ Briefly, mice were anaesthetised with $4 \%$ isoflurane and maintained with $1.5 \%$ isoflurane in an oxygen/air mixture using a gas anaesthesia mask in a stereotaxic frame (RWD Life Science, Shenzhen, China) and the body temperature was maintained at $37^{\circ} \mathrm{C}$ by using a heating pad (RWD Life Science, Shenzhen, China). 

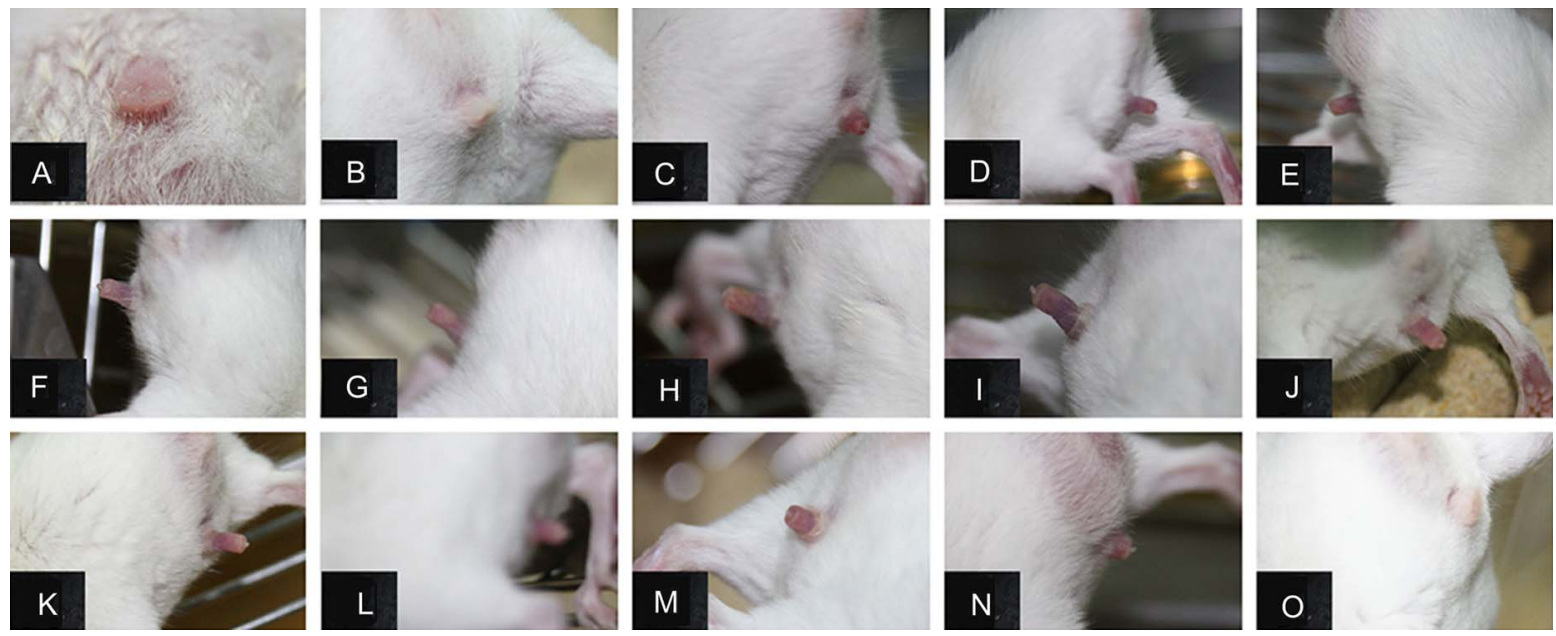

Figure 1 Adult mice were found to display priapism after permanent middle cerebral artery occlusion (pMCAO).

Photographs showed that adult mouse did not get erection before pMCAO (A), or 1 day after pMCAO (B). Persistent erection started on the 2nd day (C), maintained through the 3rd (D) to the 13th day $(\mathrm{N})$ and disappeared on the 14th day postoperation $(\mathrm{O})$.

A midline incision was made on the neck under an operating microscope (Leica, Germany). External carotid artery (ECA), common carotid artery (CCA) and internal carotid artery (ICA) were isolated. After temporarily clamping the CCA, a $6-0$ silicon-coated round-tip monofilament nylon suture was introduced into the ICA through the ECA until slight resistance was felt, indicating a complete middle cerebral artery (MCA) occlusion. Then cerebral blood flow was measured by laser Doppler flowmetry (Moor Instruments, UK) to confirm a successful MCA occlusion that has regional blood flow of $<15 \%$ of baseline blood flow.

\section{TTC staining and H\&E staining}

After sacrificing the animals, the brains were sectioned with brain matrix, and stained with a $2 \%$ solution of 2,3,5-triphenyltetrazolium (TTC) dye (Sigma, T8877$100 \mathrm{G})$ at room temperature for $20 \mathrm{~min}$ as described previously. ${ }^{18}$ Then stained sections were immersed in $4 \%$ phosphate-buffered paraformaldehyde and imaged with a digital camera. The infarct area was measured as previously described. ${ }^{19}$ Then the brains were embedded in paraffin. Coronal sections were processed and stained with H\&E (Beyotime Institute of Biotechnology). Using NIH Image 1.63 software, the ischaemic lesion area was calculated as the difference between the area of the nonischaemic hemisphere and the normal area in the ischaemic hemisphere. Infarct volume was calculated by multiplying the infarct area by the thickness of the section.

\section{Priapism recording}

The mice were observed every day after the pMCAO procedure. The time of priapism occurrence and the recovery of flaccidity (disappearance of priapism) were both recorded. Photographs were taken using a Canon digital camera (Canon EOS 450D).

\section{Statistical analysis}

The values were given as mean \pm SE. The significance of difference was calculated by one-way analysis of variance, followed by Student-Newman-Keuls post hoc comparisons. p Values $<0.05$ were considered significant.

\section{RESULTS}

\section{Priapism occurred in mice after pMCAO}

We conducted pMCAO procedure in 24 mice and found priapism occurrence in 20 of these animals. After the surgery, the duration of priapism was recorded in nine mice up to 14 days (eight mice were sacrificed for TTC staining and seven mice died after the surgery). Six mice displayed sustained penile erection from the second day after PMCAO (figure 1). The erection began to be relieved from the 7 th day to the 14th day postoperation (table 1).

Table 1 A summary of priapism condition in nine mice after permanent middle cerebral artery occlusion (pMCAO)

\begin{tabular}{lll}
\hline Number & $\begin{array}{l}\text { Erection } \\
\text { start (days) }\end{array}$ & $\begin{array}{l}\text { Erection } \\
\text { disappear (days) }\end{array}$ \\
\hline 1 & 2 & 14 \\
2 & 2 & 14 \\
3 & 2 & 7 \\
4 & $/$ & $/$ \\
5 & $/$ & $/$ \\
6 & 1 & 10 \\
7 & 2 & 9 \\
8 & $/$ & $/$ \\
9 & 1 & 11 \\
\hline
\end{tabular}

The first line means nine mice were recorded after pMCAO (number 1-9), the middle and the last line displayed when priapism had occurred and disappeared after surgery. '" Indicates no priapism was observed. 
The difference of brain infarct volume between priapism and non-priapism ischaemic mice

To compare brain infarct volume in the mice with and without priapism, the brain tissues were processed by TTC staining and H\&E staining, and the infarct area was recorded, in parallel to animals with sham operation (no ischaemia and priapism). Mice after pMCAO had brain ischaemia as demonstrated by TTC staining (left and right columns of figure 2). However, the infract volume are significantly different in mice with priapism from those without priapism, consistent with $\mathrm{H} \& \mathrm{E}$ results (figure 3). We found that in the stroke mice with priapism, the infarct area was significantly larger than those displaying no priapism (figure 4). Furthermore, the specific infarct area was also different between them. In the ischaemic mice with priapism, the hippocampus and hypothalamus were damaged by ischaemia; however, in the non-priapism mice with ischaemia, the hippocampus area and the hypothalamus area were normal (figures 2 and 3).

\section{DISCUSSION}

In this study, we demonstrated that: (1) pMCAO could induce a high occurrence of priapism in mice $(80 \%)$; (2) ischaemia-induced erection often occurs 2 days after pMCAO $(67 \%)$ and disappears between the 7 th and 14th day after pMCAO; and (3) hypothalamus and hippocampus damage may contribute to the occurrence of priapism. Our data suggested that cerebral ischaemia is closely related with priapism occurrence.

Priapism is defined as prolonged penile erection without sexual stimulation, and it often induces irreversible damage to erectile tissues and results in erection dysfunction. To the best of our knowledge, we first reported that pMCAO can induce priapism in rodents in this study. Our data showed that most ischaemic mice displayed priapism 2 days after $\mathrm{pMCAO}$, and the detumescence time varied from the 7 th day to the 14th day. As our TTC staining result revealed, there were some differences between the two groups (with and without priapism in stroke mice). The infarct volume in the mice with priapism was much larger, and the infarct area included the hypothalamus and hippocampus. However, these areas in the non-priapism mice were normal, suggesting that priapism is closely related to the lesion site of stroke, especially related to hippocampus and hypothalamus. We propose these differences are caused by posterior communicating artery (PComA) variation, which supplies blood to the hippocampus and hypothalamus regions. ${ }^{20}$ Our results might also indicate that hypothalamus and hippocampus injury are responsible for priapism. Indeed, these results are consistent with some previous studies. Some researchers have demonstrated that stimulation of brain can induce penile erection, and hippocampus and hypothalamus are important erection mediators. ${ }^{21}$ Melis et $a l^{22}$ reported that microinjection of oxytocin into the paraventricular
Figure 2 Priapism closely correlated with infarct area. Photographs showed that the sham group did not get priapism and infarct in the brain (middle column, B-K); ischaemic mice that also displayed priapism were found to have extensive infarct affecting both hypothalamus (arrowhead) and hippocampus (arrow) areas (right column, C-L); in contrast, ischaemic mice that did not display priapism were found to have non-infarct hypothalamus and hippocampus areas (left column, A-J). All mice were sacrificed 3 days after permanent middle cerebral artery occlusion, $n=4$ for each group.
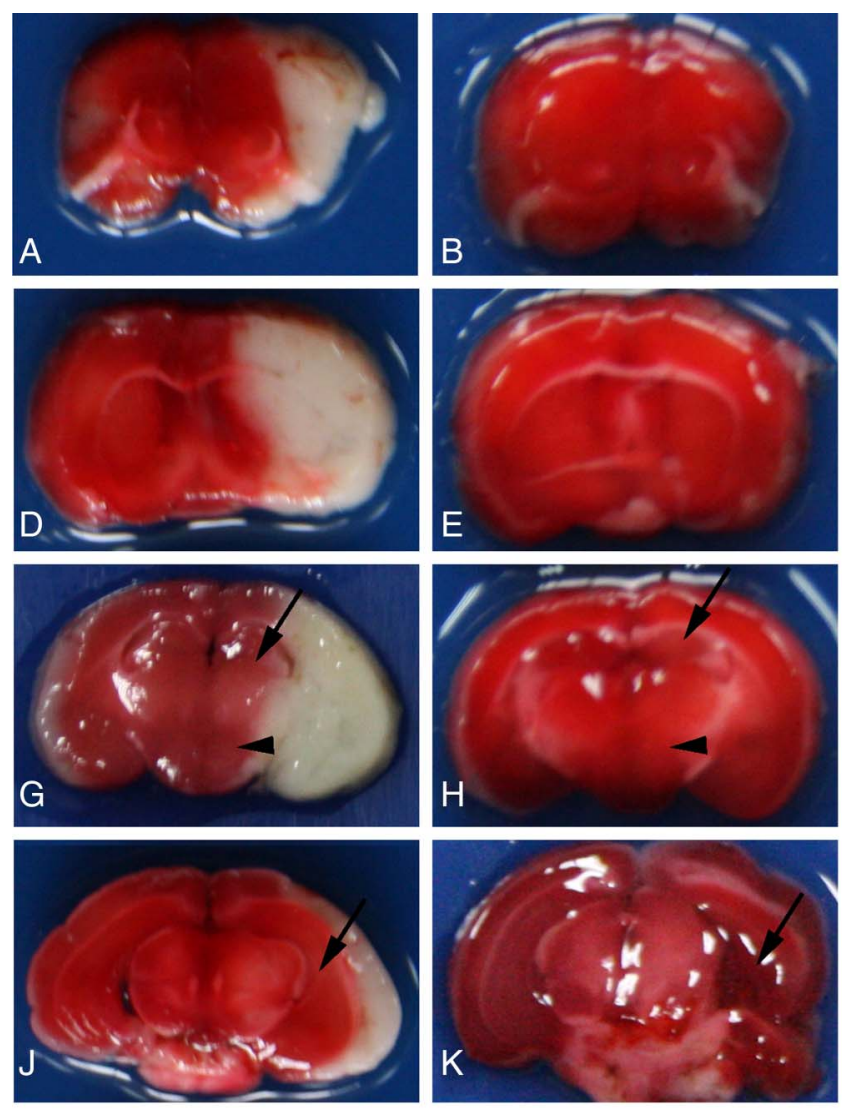

Ischaemia and non-priapism

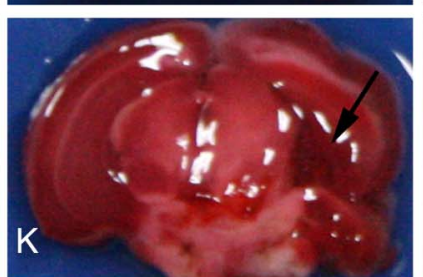

Sham
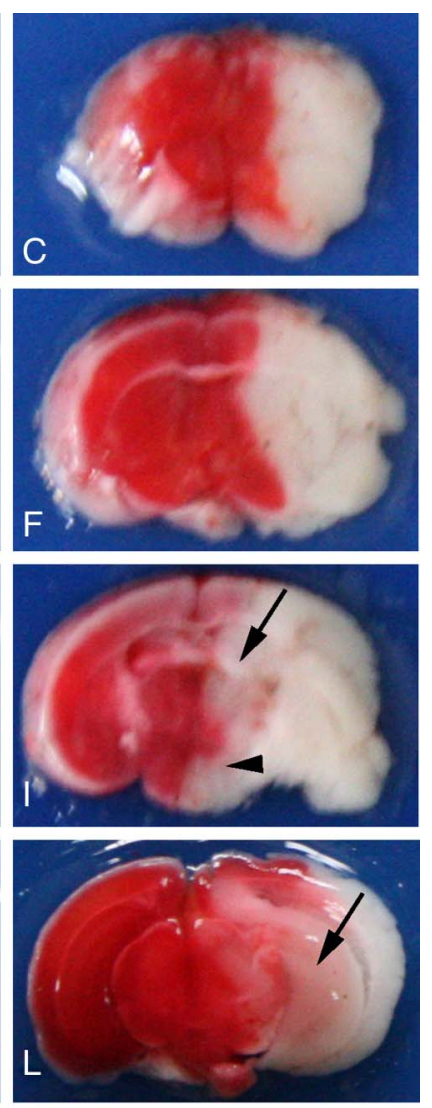

Ischaemia and priapism 
Figure 3 Priapism occurrence was related with different infarct area. H\&E staining showed that the sham group did not show priapism, and the nuclei of cells in these brains were normal $(B$ and $E$ ); in the group of ischaemic mice with priapism ( $\mathrm{C}$ and $\mathrm{F}$ ), the nuclei of the cells in the hippocampus (arrow head) and hypothalamus (white arrow) displayed shrinkage; however, in the group of ischaemic mice without priapism (A and $D)$, the hippocampus (arrowhead) and hypothalamus (white arrow) showed normal nuclei size; photographs A, C, D, F were taken from a, c, d and f, respectively. Bar $=20 \mu \mathrm{m}, \mathrm{n}=4$ for each group.

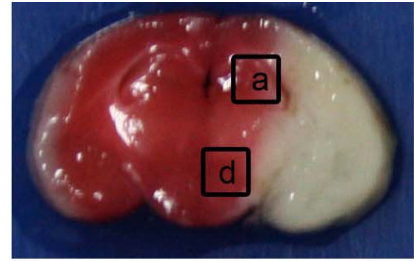

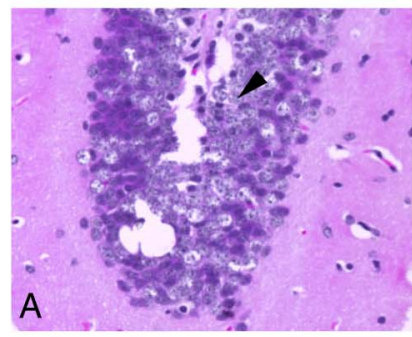

Hippocampus (stroke with nonpriapism)

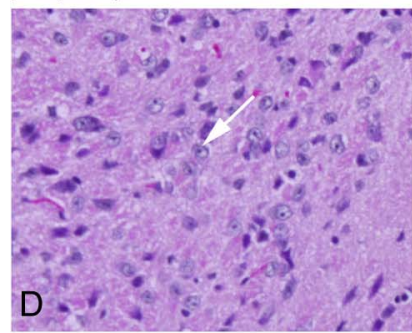

Hypothalamus (stroke with nonpriapism)

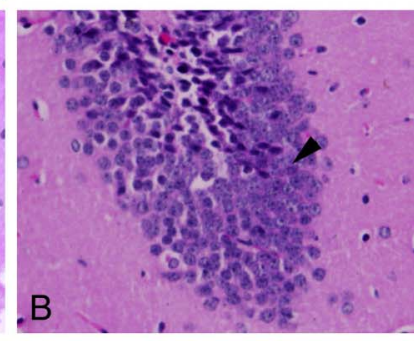

Hippocampus (sham)

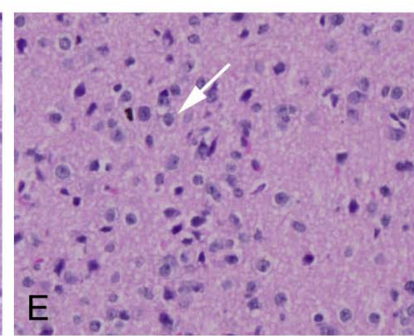

Hypothalamus (sham)
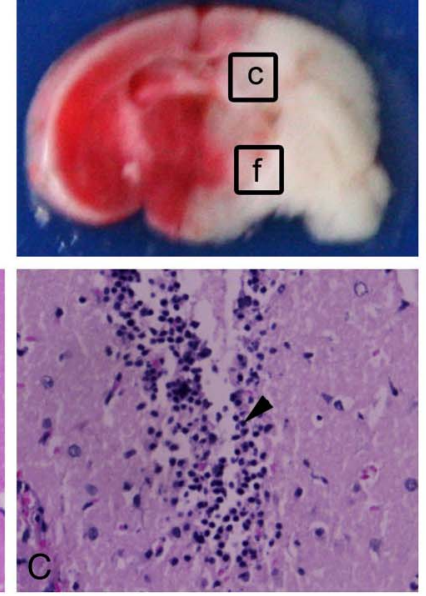

Hippocampus (stroke with priapism)

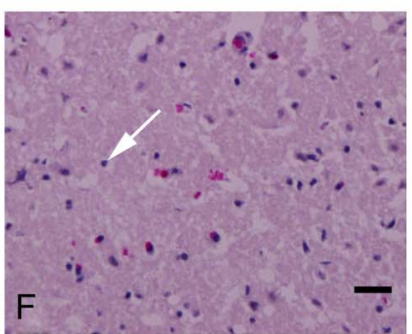

Hypothalamus (stroke with priapism)

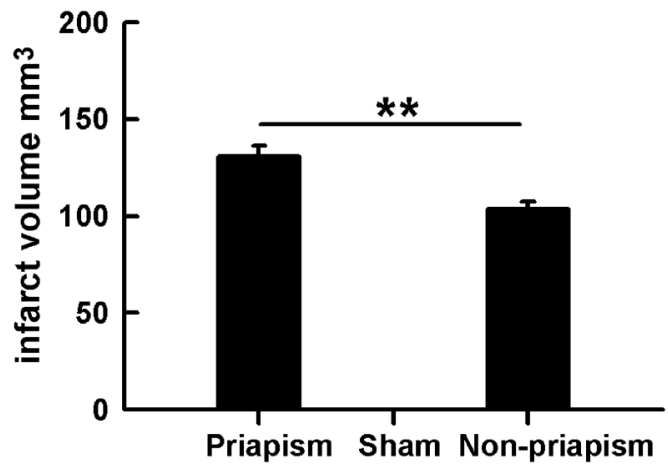

Figure 4 The infarct volume was significantly larger in stroke mice with priapism than those without priapism. Bar graph showed significant difference of infarct volume between the stroke priapism mice and stroke non-priapism mice. ${ }^{\star *} \mathrm{p}<0.01$, $n=4$ for each group.

nucleus of the hypothalamus or into the CA1 field of the hippocampus induced penile erection in male rats. Arrow suggested that penile erection was associated with brain region activation, including the claustrum, left caudate and putamen, right middle occipital/middle temporal gyri, bilateral cingulated gyrus, right sensorimotor, premotor regions and right hypothalamus. ${ }^{23}$ Other studies showed that anaesthesia could also induce priapism. For instance, Senthilkumaran et $a t^{24}$ reported that propofol induced priapism in a 25-year-old man, Ravindran et $a l^{25}$ reported two cases of priapism induced by $0.5 \% \mathrm{mg} / \mathrm{kg}$ ketamine and $1.5 \mathrm{mg}$ of physostigmine.
However, in these clinical cases, the reasons why priapism responds to anaesthesia are not explored.

To further investigate the association between the brain region and priapism, functional MRI may be required in follow-up studies. Besides the damage to the specific brain region, we suggest that priapism can be related to some chemicals induced by stroke, such as nitric oxide (NO), adenosine and so on. These chemicals are closely related with stroke, and they also play important roles in erection. $\mathrm{NO}$ is reported to be an important factor in stroke ${ }^{26}$ and penile erection. ${ }^{27}$ It is closely associated with stroke and shows beneficial actions, ${ }^{28}$ including enhancing angiogenesis via the synthesis of vascular endothelial growth factor and cyclic guanosine monophosphate after stroke. ${ }^{29} \mathrm{NO}$ is also an important mediator in penile erection. Azadzoi et $a b^{30}$ stated that neuronal nitric oxide synthase (nNOS), endothelial nitric oxide synthase (eNOS) and inducible nitric oxide synthase (iNOS) levels in cavernosum were upregulated after atherosclerosis-induced ischaemia, and NO-mediated smooth muscle relaxation in a rabbit. However, we peritoneally injected the NOS inhibitor LNMMA (Beyotime, S0011) into the stroke mice, priapism did not disappear (data not shown). We cannot fully exclude the possible involvement of NO in causing priapism by this simple inhibitor study though. Further experiments are needed to clarify its role in the phenomenon reported here. Adenosine is an inhibitory modulator of brain activity with neuroprotective properties, ${ }^{31}$ and recent studies have reported that adenosine 
contributed to animal priapism. ${ }^{9}$ Intracavernous injection of adenosine resulted in tumescence and penile erection. ${ }^{32}$ Adenosine accumulated in the penis coupled with its receptor A2BR, contributed to priapism, ${ }^{33}$ and ophylline, an adenosine receptor antagonist, inhibited adenosine-induced penile tumescence. ${ }^{34}$ However, whether adenosine is a potential agent which mediates both stroke and priapism needs to be further discussed. Recent studies showed that in corpus cavernosum ischaemia-reperfusion mouse, many oxidative injury parameters like superoxide dismutase (SOD), catalase, malondialdehyde (MDA) and NO in the tissues were all upregulated, and melatonin, which also played neuroprotective actions in stroke, ${ }^{35}$ could attenuate the activity of SOD and the levels of MDA and NO. ${ }^{36}$ These conclusions strongly suggested that melatonin may be involved in the stroke-induced priapism pathway. If these chemicals are indeed associated with stroke and priapism, drugs used to cure priapism may also interfere in signalling pathways in stroke and some of them could possibly show beneficial effects to the ischaemic brain. This line of study might also be worth exploring.

In conclusion, this investigation has demonstrated the correlation between stroke and priapism. Most mice got sustained erection after pMCAO though their hypothalamus and hippocampus area were damaged. It is conceivable that priapism may serve as a physiological marker for adult stroke mouse. Since the patency of PComA plays an important role in the infarct results of the murine pMCAO models, ${ }^{37}$ an effective evaluation method is urgently needed to reduce the fluctuation of the infarct area among different individual animals. ${ }^{38}$ It is likely that we can use priapism as a physiological marker to identify stable pMCAO models more conveniently, which may facilitate the study of stroke.

\section{CONCLUSION}

Our study demonstrated that the occurrence of priapism is related to the infarct region: priapism is found only in mice with ischaemic injury extending to the hypothalamus and the hippocampus regions. Our results suggested priapism may be used as a deep brain injury marker for evaluating brain injury in mice after pMCAO.

Acknowledgements The authors thank Caibin Sheng for his help taking the photographs, Minjie Shen for editorial assistance and the staff of Neuroscience and Neuroengineering Center for their collaborative support.

Contributors $\mathrm{YT}, \mathrm{FY}$ and $\mathrm{BC}$ participated in the experimental design and data analysis. They prepared animal ischaemia model, analysed the data and drafted the manuscript. WX and YW participated in the experimental design and manuscript discussion. G-YY took care of all aspects, including participating in the study design, supervising tissue assays, analysing the results, and organising and finalising the manuscript.

Funding The study is supported by the National Natural Science Foundation of China, 81471178(GYY), U1232205 (GYY) and 81371305 (YW).

Competing interests None declared.

Provenance and peer review Commissioned; externally peer reviewed.
Data sharing statement No additional data are available.

Open Access This is an Open Access article distributed in accordance with the Creative Commons Attribution Non Commercial (CC BY-NC 4.0) license, which permits others to distribute, remix, adapt, build upon this work noncommercially, and license their derivative works on different terms, provided the original work is properly cited and the use is non-commercial. See: http:// creativecommons.org/licenses/by-nc/4.0/

\section{REFERENCES}

1. Rosamond W, Flegal K, Furie K, et al. Heart disease and stroke statistics-2008 update: a report from the American Heart Association Statistics Committee and Stroke Statistics Subcommittee. Circulation 2008;117:e25-146.

2. Chan $\mathrm{PH}$. Role of oxidants in ischemic brain damage. Stroke 1996;27:1124-9.

3. Ankarcrona M, Dypbukt JM, Bonfoco E, et al. Glutamate-induced neuronal death: a succession of necrosis or apoptosis depending on mitochondrial function. Neuron 1995;15:961-73.

4. Huang J, Li Y, Tang Y, et al. CXCR4 antagonist AMD3100 protects blood-brain barrier integrity and reduces inflammatory response after focal ischemia in mice. Stroke 2013;44:190-7.

5. Montague DK, Jarow J, Broderick GA, et al. American Urological Association guideline on the management of priapism. J Urol 2003;170:1318-24

6. Serjeant GR, de Ceulaer K, Maude GH. Stilboestrol and stuttering priapism in homozygous sickle-cell disease. Lancet 1985;2: 1274-6.

7. El-Bahnasawy MS, Dawood A, Farouk A. Low-flow priapism: risk factors for erectile dysfunction. BJU Int 2002;89:285-90.

8. Kanika ND, Tar M, Tong $\mathrm{Y}$, et al. The mechanism of opiorphin-induced experimental priapism in rats involves activation of the polyamine synthetic pathway. Am J Physiol Cell Physiol 2009;297:C916-27.

9. Wen J, Jiang $X$, Dai $Y$, et al. Increased adenosine contributes to penile fibrosis, a dangerous feature of priapism, via $A 2 B$ adenosine receptor signaling. FASEB J 2010;24:740-9.

10. Takaku A, Fukawa $O$, Suzuki J. A case of priapism with ruptured intracranial aneurysm. J Neurol 1979;221:279-83.

11. Monga TN, Monga M, Raina MS, et al. Hypersexuality in stroke. Arch Phys Med Rehabil 1986;67:415-17.

12. Fan $Y$, Shen F, Frenzel T, et al. Endothelial progenitor cell transplantation improves long-term stroke outcome in mice. Ann Neurol 2010;67:488-97.

13. Yang G, Chan $\mathrm{PH}$, Chen J, et al. Human copper-zinc superoxide dismutase transgenic mice are highly resistant to reperfusion injury after focal cerebral ischemia. Stroke 1994;25:165-70.

14. Yang GY, Gong C, Qin Z, et al. Inhibition of TNFalpha attenuates infarct volume and ICAM-1 expression in ischemic mouse brain. Neuroreport 1998;9:2131-4.

15. Yuan $\mathrm{F}$, Tang $\mathrm{Y}$, Lin $\mathrm{X}$, et al. Optimizing suture middle cerebral artery occlusion model in C57BL/6 mice circumvents posterior communicating artery dysplasia. J Neurotrauma 2012;29:1499-505.

16. Lin X, Miao P, Wang J, et al. Surgery-related thrombosis critically affects the brain infarct volume in mice following transient middle cerebral artery occlusion. PLOS ONE 2013;8:e75561.

17. Zhang $\mathrm{L}$, Wang $\mathrm{Y}$, Tang $\mathrm{Y}$, et al. High MRI performance fluorescent mesoporous silica-coated magnetic nanoparticles for tracking neural progenitor cells in an ischemic mouse model. Nanoscale 2013;5:4506-16.

18. Bederson JB, Pitts LH, Germano SM, et al. Evaluation of 2,3,5-triphenyltetrazolium chloride as a stain for detection and quantification of experimental cerebral infarction in rats. Stroke 1986;17:1304-8.

19. Lin TN, He YY, Wu G, et al. Effect of brain edema on infarct volume in a focal cerebral ischemia model in rats. Stroke 1993;24:117-21.

20. Dawson $\mathrm{BH}$. The blood vessels of the human optic chiasma and their relation to those of the hypophysis and hypothalamus. Brain 1958;81:207-17.

21. Argiolas A, Melis MR. Central control of penile erection: role of the paraventricular nucleus of the hypothalamus. Prog Neurobiol 2005;76:1-21.

22. Melis MR, Argiolas A, Gessa GL. Oxytocin-induced penile erection and yawning: site of action in the brain. Brain Res 1986;398:259-65.

23. Arnow BA, Desmond JE, Banner LL, et al. Brain activation and sexual arousal in healthy, heterosexual males. Brain 2002;125:1014-23.

24. Senthilkumaran S, Shah S, Ganapathysubramanian, et al. Propofo and priapism. Indian J Pharmacol 2010;42:238-9. 
25. Ravindran RS, Dryden GE, Somerville GM. Treatment of priapism with ketamine and physostigmine. Anesth Analg 1982;61:705-7.

26. Samdani AF, Dawson TM, Dawson VL. Nitric oxide synthase in models of focal ischemia. Stroke 1997;28:1283-8.

27. Burnett AL, Lowenstein CJ, Bredt DS, et al. Nitric oxide: a physiologic mediator of penile erection. Science 1992;257:401-3.

28. Ignarro LJ. Nitric oxide as a unique signaling molecule in the vascular system: a historical overview. J Physiol Pharmacol 2002;53:503-14.

29. Zhang R, Wang L, Zhang $L$, et al. Nitric oxide enhances angiogenesis via the synthesis of vascular endothelial growth factor and cGMP after stroke in the rat. Circ Res 2003;92:308-13.

30. Azadzoi KM, Master TA, Siroky MB. Effect of chronic ischemia on constitutive and inducible nitric oxide synthase expression in erectile tissue. J Androl 2004:25:382-8.

31. Boison D. Adenosine kinase, epilepsy and stroke: mechanisms and therapies. Trends Pharmacol Sci 2006;27:652-8.

32. Chiang PH, Wu SN, Tsai EM, et al. Adenosine modulation of neurotransmission in penile erection. Br J Clin Pharmacol 1994;38:357-62.
33. Dai $\mathrm{Y}$, Zhang $\mathrm{Y}$, Phatarpekar $\mathrm{P}$, et al. Adenosine signaling, priapism and novel therapies. J Sex Med 2009;6(Suppl 3):292-301.

34. Noto $\mathrm{T}$, Inoue $\mathrm{H}$, Mochida $\mathrm{H}$, et al. Role of adenosine and $\mathrm{P} 2$ receptors in the penile tumescence in anesthetized dogs. Eur J Pharmacol 2001;425:51-5.

35. Reiter RJ, Sainz RM, Lopez-Burillo S, et al. Melatonin ameliorates neurologic damage and neurophysiologic deficits in experimental models of stroke. Ann N Y Acad Sci 2003;993:35-47; discussion 48-53.

36. Uluocak $\mathrm{N}$, Atilgan $\mathrm{D}$, Erdemir $\mathrm{F}$, et al. An animal model of ischemic priapism and the effects of melatonin on antioxidant enzymes and oxidative injury parameters in rat penis. Int Urol Nephrol 2010;42:889-95.

37. Kitagawa K, Matsumoto M, Yang G, et al. Cerebral ischemia after bilateral carotid artery occlusion and intraluminal suture occlusion in mice: evaluation of the patency of the posterior communicating artery. J Cereb Blood Flow Metab 1998;18:570-9.

38. Belayev L, Busto R, Zhao W, et al. Middle cerebral artery occlusion in the mouse by intraluminal suture coated with poly-L-lysine: neurological and histological validation. Brain Res 1999;833:181-90. 